\title{
Advances and Challenges in Intensity-Modulated Radiotherapy for Nasopharyngeal Carcinoma
}

\author{
Song Qu, Zhong-Guo Liang, Xiao-Dong Zhu*
}

\begin{abstract}
Nasopharyngeal carcinoma is an endemic disease within specific regions in the world. Radiotherapy is the main treatment. In recent decades, intensity-modulated radiation therapy has undergone a rapid evolution. Compared with two-dimensional radiotherapy and/or three-dimensional conformal radiotherapy, evidence has shown it may improve quality of life and prognosis for patients with nasopharyngeal carcinoma. In addition, helical tomotherapy is an emerging technology of intensity-modulated radiation therapy. Its superiority in dosimetric and clinical outcomes has been demonstrated when compared to traditional intensity-modulated radiation therapy. However, many challenges need to be overcome for intensity-modulated radiation therapy of nasopharyngeal carcinoma in the future. Issues such as the status of concurrent chemotherapy, updating of target delineation, the role of replanning during IMRT, the causes of the main local failure pattern require settlement. The present study reviews traditional intensity-modulated radiation therapy, helical tomotherapy, and new challenges in the management of nasopharyngeal carcinoma.
\end{abstract}

Keywords: Nasopharyngeal carcinoma - intensity-modulated radiotherapy - two-dimensional radiotherapy

Asian Pac J Cancer Prev, 16 (5), 1687-1692

\section{Introduction}

Nasopharyngeal carcinoma (NPC) is an endemic disease within specific regions in the world. Incidence rates of NPC are high in South-Eastern Asia, including Malaysia, Indonesia, Singapore, and South-Eastern China (Jemal et al., 2011).

Radiation therapy is the main treatment for NPC. In 2006, Yi et al. (2006) reported on their experience in the treatment of NPC by conventional radiotherapy alone in their institution during the last decade. The 5-year overall survival (OS) rates were reported to be about $95.5 \%$ and $87.7 \%$ for stageIand II NPC, while they were $76.9 \%$ and $66.39 \%$ for stage III and IV disease.

Intensity-modulated radiotherapy (IMRT) is a major breakthrough in the treatment of NPC. It possesses the superiority in excellent target coverage and normal tissue sparing when compared to two-dimensional radiotherapy (2DRT) and three-dimensional conformal radiotherapy (3DCRT) (Chau et al., 2007; Phua Chee Ee et al., 2013). Recent trials showed that IMRT could not only improve the quality of life but also significantly improve survival (Lai et al., 2011; Peng et al., 2012).

Helical tomotherapy, as an emerging technology of IMRT, had been approved an innovative radiation therapy device by the United States Food and Drug Administration (FDA) in 2002. Studies have reported that compared to traditional IMRT, it achieved better target coverage with improved organ at risk (OAR) sparing (Rong et al., 2011; Wiezorek et al., 2011). What's more, there exist great challenges for IMRT in the therapy of NPC. For example, in 2013, Chen et al, (2013) calculate dosimetric changes of target volumes and OAR in NPC during IMRT. They concluded that weight loss may lead to significant dosimetric change of gross target volume (GTV) and OAR during IMRT. It need to be solved whether it is necessary to repeat scanning and replan the radiotherapy for NPC and what time is appropriate for replannning.

This article examines the pertinent issues and latest studies concerning traditional IMRT, helical tomotherapy, and new challenges for IMRT in the management of NPC.

\section{Intensity-Modulated Radiotherapy}

\section{Technical Advances}

For NPC, the tumor is approximately close to the critical organ such as spinal cord, brainstem, and so on. In order to protect the critical normal organs, there exists a limitation in delivering high dose to the targets for 2DRT. IMRT which delivers a high dose of radiation to the tumor while keeping a reduced dose to normal tissues surrounding the NPC region and excellent tumor coverage (Chau et al., 2007; Lin et al., 2009; Tham et al., 2009; Phua Chee Ee et al., 2013). Chau et al. (2007) conducted a study to evaluate target coverage and organ protection between the technology of 2DRT and IMRT in the treatment of 
advanced stage T3-4 NPC. They found the D95\% of the GTV and plan target volume (PTV) were increased from $57.1 \mathrm{~Gy}$ (2DRT) to $67 \mathrm{~Gy}$ (IMRT), and $45 \mathrm{~Gy}$ (2DRT) to 63.6 Gy (IMRT), respectively. The mean maximum dose delivered to the brainstem and spinal cord were reduced significantly from 61.8 Gy (2DRT) to 52.8 Gy (IMRT) and 56 Gy (2DRT) to 43.6 Gy (IMRT), respectively. In 2013, Phua et al (Phua Chee Ee et al., 2013) compared the dosimetric coverage of target volumes and organs at risk for NPC between IMRT and three-dimensional conformal radiotherapy (3DCRT). Data of Ten patients were retrospectively analyzed. Target volume coverage of V70, V66.5, V59.4 and V56.4 for PTV70 showed marked dosimetric superiority of IMRT over 3DCRT. What's more, IMRT was superior in sparing the spinal cord, parotid and the left cochlea than 3DCRT.

\section{Quality of life}

Dosimetric superiority of IMRT over 2DRT could translate into improved quality of life (QoL) for patients with NPC. From March 2003 to May 2004, 16 patients with nonmetastatic NPC underwent parotid-sparing IMRT (Hsiung et al., 2006). The parotid function of 16 patients who were previously treated with 2DRT was reviewed as the historical control. The post-IMRT parotid function was evaluated by quantitative salivary scintigraphy and represented by the maximal excretion ratio (MER) of the parotid gland after sialogogue stimulation. The mean parotid MER was $53.5 \%$ before radiotherapy, $10.7 \%$ at 1 month post-IMRT, and $23.3 \%$ at 9 months post-IMRT. In the 2DRT group, the mean parotid MER was $0.6 \%$ at 6 to 12 months postradiotherapy. The difference was statistically significant $(\mathrm{P}<0.001)$. Ma et al (Sun et al., 2013) performed a cross-sectional study to compare the changing tendency of QoL between patients who received IMRT and 2DRT with different follow-up time using an NPC-specific QoL instrument, and further validate the advantage of IMRT in QoL of NPC. 75 patients were treated with IMRT and 67 received 2DRT. At 12-18 months after treatment, all QoL scores of patients were comparable in both groups. At 19-28 months, a statistically significant improvement of QoL in symptom scales in the IMRT group was observed when compared with that of the 2DRT group $(78.48 \pm 9.30$ vs $69.66 \pm 12.03, \mathrm{P}=0.008)$. At 29-42 months, QoL scores of physical functioning scales, global health status and symptom scales were significantly superior in the IMRT group $(90.72 \pm 9.87$, $83.16 \pm 13.65$ and $66.67 \pm 23.57$, respectively) than those in the 2DRT group $(50.00 \pm 31.47,78.46 \pm 11.79$ and $67.85 \pm 13.86$, respectively). Compared with 2DRT, IMRT substantially improved QoL in NPC patients. And the superiority became significant with prolonged followup time. However, it was unknown whether NPC could benefit significant QOL from IMRT when compared to three-dimensional conformal radiotherapy (3DCRT). In order to answer this question, 243 newly diagnosed NPC patients, who were curatively treated by 3DCRT $(n=93)$ or IMRT ( $\mathrm{n}=110)$ were analyzed (Fang et al., 2008). In this study, QoL was longitudinally assessed by the European Organization for Research and Treatment of Cancer (EORTC) QLQ-C30 and the EORTC QLQ-H\&N35 questionnaires at the five time points: before radiotherapy, during radiotherapy ( $36 \mathrm{~Gy}$ ), and 3 months, 12 months, and 24 months after radiotherapy. This study showed that there were no significant differences in most scales between the two groups at each time point. Trials with large samples were essential to performed to determine the superiority of IMRT to 3DRT.

What's more, IMRT could decline incidences of osteoradionecrosis of the external auditory canal in NPC when compared to 2DRT. In 2012, Tsang et al. (2012) compared long-term hearing results and ontological complications of NPC between the IMRT group and the 2DRT group. The results showed that there was a significant deterioration of the hearing threshold 5 years after radiotherapy between IMRT and 2DRT. 6 patients in the 2DRT group and 1 patient in the IMRT group had osteoradionecrosis of the external auditory canal $(\mathrm{P}=0.042)$.

\section{Survival benefit}

IMRT creates steep dose gradient at the border between the target and adjacent normal tissues. Inaccurate target delineation will result in either underdose to target or overdose to normal tissues, or both, which may subsequently influence tumor control in the management of NPC. Compared to 2DRT, would IMRT provide survival benefit for locally advanced NPC? In Peng et al's trial (Peng et al., 2012), 616 patients with non-metastatic stage I-IVb NPC were randomly assigned to receive 2DRT $(n=310)$ or IMRT $(n=306)$. After a median follow-up of 42 months, the 5-year OS rate was $79.6 \%$ vs $67.1 \%$ for the IMRT group and the 2DRT group ( $\mathrm{P}=0.001)$. A retrospective analysis was performed to evaluate the efficacy of IMRT in primary NPC patients compared with 2DRT (Zhang et al., 2009). 190 patients with NPC treated with IMRT and another 190 patients treated with 2DRT at the same period were analyzed. With a median follow up of 39 months, there were no significant differences in the 4-year OS between the two groups. However, from the OS curves between the two groups, there was a significantly trend of improvement of OS in the IMRT group compared with the 2DRT group. This might be associated with the time of follow-up. However, in Peng et al's trial (Peng et al., 2012), there were only $34.3 \%$ (105/306) and 33.2\% $(103 / 310)$ patients received concurrent chemotherapy in the IMRT group and the 2DRT group. In Zhang et al's trial (Zhang et al., 2009), only $4.2 \%$ (8/190) patients received concurrent chemotherapy in the IMRT group or the 2DRT group. It was essential to conduct multicenter controlled trials with larger samples and long follow-up to evaluate whether patients with NPC could acquire more survival benefit from IMRT than 2DRT in the mode of standard therapeutic regimen recommended by the National Comprehensive Cancer Network (NCCN).

Lai et al. (2011) conducted a retrospective study of 1,276 patients with biopsy-proven, non-metastatic NPC to compare the results of IMRT with 2DRT. Of the 1,276 patients, 512 were treated with IMRT and 764 with 2DRT. The 5-year actuarial local relapse-free survival (LRFS), the nodal relapse-free survival (NRFS), the distant metastasis failure-free survival (DMFS), and the 
disease free survival (DFS) rates were 92.7\%, 97.0\%, $84.0 \%$, and $75.9 \%$, respectively, for the IMRT group, and $86.8 \%, 95.5 \%, 82.6 \%$, and $71.4 \%$, respectively, for the 2DRT group. They concluded that a greater improvement of treatment results with IMRT than with 2DRT was demonstrated primarily by achieving a higher local tumor control rate in NPC patients, especially in the early T stage patients.

In 2014, Lee et al. (2014) reported a retrospective study which assessed the therapeutic gains as the technology evolved from 2DRT to 3DCRT and to IMRT era for NPC. 434 patients in the 2DRT group, 715 patients in the 3DCRT group, and 444 patients in the IMRT group were analyzed. The median follow-up of the whole series was 6.8 years. Compared to 2DRT, significant improvements were achieved in 5-year local failure-free rate for T3-4 patients in the IMRT group. What's more, IMRT was superior to 2DRT in 5-year distant failure-free rate, disease-specific survival, and OS for stage III-IV disease. However, IMRT only showed significant advantage in 5-year OS, but not in 5-year local failure-free rate, distant failure-free rate, and disease-specific survival when compared with 3DCRT. In another study, 52 patients were reviewed to compare IMRT versus 2DRT/3DCRT in the therapy of NPC (Moretto et al., 2014). After a median follow-up of 37.6 months, they found that IMRT was not significantly superior to 2DRT/3DCRT in two years local control rate, locoregional control rate, DFS, and OS. However, the sample of this study was small. RCTs with large samples need to be conducted to research whether IMRT can supply significant survival benefit for NPC when compared to 3DCRT.

\section{Helical tomotherapy}

Helical tomotherapy is an emerging technology of IMRT. It combines the linear accelerator and spiral computed tomography (CT) scan together. The patient would move through the bore of the gantry simultaneously with gantry rotation. The intensity modulation would be performed by temporally modulated multiple independent leaves that open and close across the slit opening (Mackie et al.,1993). Compared to the traditional linear accelerator, it is free from the limitation of incident angle. Therefore, it can deliver a high dose of radiation to the target while keeping a reduced dose to normal tissues. Wu et al ( $\mathrm{Wu}$ et al., 2010) compared the dosimetric outcomes between the tomotherapy plans and conventional IMRT plans in the treatment of NPC. Both the tomotherapy plan and 7-field 6-MV photon conventional IMRT plan were computed for 15 stage II-III NPC at the same time. In the tomotherapy plan, the differences in the maximum and mean doses of PTV were statistically significant $(p<0.05)$. What's more, the conformity index of the tomotherapy was significantly higher and the homogeneity index was significantly smaller in for the nasopharynx PTV and neck lymphatics PTV than the conventional IMRT plans. The brain stem and spinal cord received lower doses in the tomotherapy plans. In 2014, Lee et al. (2014) found that tomotherapy achieved significantly better dose conformity to the treatment targets and delivered significantly lower doses in lens and mandible than sliding-window IMRT.
Several studies have reported survival benefit and toxicities of helical tomotherapy in therapy of NPC. In 2011, Ren et al (Ren et al., 2011) reported their clinical observation of 73 nasopharyngeal carcinoma patients treated by helical tomotherapy. 24 patients were treated with radiation therapy alone, 25 with concurrent cisplatin-based chemotherapy with or without target therapy, and 24 with concurrent target therapy. The incidence of severe acute toxicities and late xerostomia was relatively infrequent for NPC patients treated with helical tomotherapy. After a median follow-up of 14.8 months, the one-year relapse-free survival (RFS), DMFS and OS was $95.6 \%, 97.2 \%$ and $94.8 \%$, respectively. Chen et al (Chen et al., 2012) compared differences in dosimetric, clinical and QoL end points among patients treated with helical tomotherapy and segmental multileaf collimator (SMLC) IMRT. 14 patients were treated using helical tomotherapy and 16 were treated using SMLCbased IMRT. After a median follow-up of 30 months, no significant differences were found in 2-year OS, locoregional failure-free survival (LFFS), and progressionfree survival (PFS) between helical tomotherapy and SMLC-based IMRT. With respect to mean radiation dose, helical tomotherapy had significantly improved salivary sparing. The corresponding proportion of patients who subjectively reported "too little" or "no" saliva at final follow-up was 38\% and 7\% among patients treated with SMLC-based IMRT and helical tomotherapy, respectively ( $p=0.04)$. In 2014, Du et al. (2014) also reported their experiences in treating NPC with helical tomotherapy. 190 newly diagnosed NPC patients treated with helical tomotherapy were included. 31 patients were treated with radiation therapy alone, 129 with additional cisplatinbased chemotherapy with or without target therapy, and 30 with concurrent target therapy. Acute radiation related side effects were mainly grade 1 or 2 . With a median follow up of 32 months, the 3-year LRFS, NRFS, DMFS, and OS were $96.1 \%, 98.2 \%, 92.0 \%$, and $86.3 \%$, respectively.

However, in Ren et al's (Ren et al., 2011) and Chen et al's (Chen et al., 2012)studies, the sample were both small. And Ren et al's (Ren et al., 2011) and Du et al's trial (Du et al., 2014) were uncontrolled studies. What's more, the follow-up term was short in these three studies. Therefore, it is urgent to conduct RCTs with large samples and long-term follow up to compare helical tomotherapy and traditional IMRT.

\section{Current Challenges and Future Directions}

Although great advances have been achieved in radiation technology, there are some challenges which need to overcome in the future. With IMRT, it's unknown whether it is still essential to use concurrent chemotherapy for loco-regional advanced NPC. Sun et al (2014) reported a retrospective study which evaluated long-term survival outcomes and toxicity of NPC patients treated with IMRT. A total of 868 non-metastatic NPC were included. 603 patients with stage III-IVb disease (according to 2002 UICC stage system) were divided into two groups: with or without concurrent chemotherapy. After a median followup of 50 months, no survival benefits were found from the 
addition of concurrent chemotherapy to IMRT in 5-year disease specific survival (DSS), LRFS, NRFS, DMFS, and PFS. What's more, patients with concurrent chemotherapy not only had higher incidence of acute toxicity such as severer mucositis, xerostomia and tympanitis, but also had higher incidence of late otologic toxicities than patients without concurrent chemotherapy. In Lin et al's trial (Lin et al., 2010), 370 patients with locoregionally advanced NPC were treated with IMRT. With a median follow-up of 31 months, subgroup analysis showed that concurrent chemotherapy supplied no significant benefit to IMRT for locoregionally advanced NPC, and it resulted higher rates of grade 3 or 4 acute toxicities. In future, an ongoing RCT may help solve this issue (NCT01817023)

The current delineation protocols regarding the target delineation are largely derived from experiences of 3DCRT and 2DRT. With the superiority of IMRT, the present delineation of clinical target volume (CTV) might be large. Guidelines regarding including CTV and many critical organs at risk might be necessary to be updated. In 2014, Lin et al (Lin et al., 2014) reported the feasibility of defining CTV of the primary lesion through GTV expansion to a minimally required margin in different directions in NPC. After a median follow-up of 60 months, 398 cases without metastasis were analyzed. The 5-year estimated OS, DFS, and local control were $80 \%, 77 \%$ and $95 \%$, respectively. No significant differences were found in margins in all the 6 directions between the 15 patients who developed local recurrence and the whole patients. For stage T4 disease, margins in all the 6 directions were significantly smaller than that of the whole group of patients. Therefore, they concluded using GTV+margin (used in T4 patients)+the whole nasopharyngeal mucosa as the definition, especially for the patients with early $\mathrm{T}$ disease, might be feasible. Sun et al (2014) compared volumes and dosimetric parameters of two to four contouring methods for the middle ear, inner ear, temporal lobe, parotid gland and spinal cord which were identified in published studies. They recommended the boundaries of these organs based on anatomic definitions and the pathogenesis of radiation-induced injury.

Replanning might be necessary for NPC during IMRT. $\mathrm{Lu}$ et al. (2014) evaluated anatomical and dosimetric changes during IMRT for NPC. Plan1 of radiotherapy was based on the original CT, and Plan2 was generated from the midtreatment CT scan when 25 fractions of Plan1 were finished. Significant changes were found in the transverse diameter of the neck and the mean volumes of the right and left parotid glands. And they found if replanning was not made, the doses to targets would be decreased, and the doses to normal tissue were increased. In 2013, Yang et al. (2013) assessed whether replanning could make effects on QoL and survival during IMRT for NPC. 43 patients received IMRT without replanning, while 86 patients received IMRT with replanning. The replanning group achieved significant higher 2-year local regional control and similar 2-year OS. In addition, they enjoyed a profound benefit in QoL from replanning. Yan et al. (2013)conducted a prospective study to explore what time is appropriate for replanning through weekly repeat computed tomography (CT) imaging during the course of IMRT. Twenty patients with stage III - Iva NPC were analyzed. The greatest reductions in GTV and CTV occurred at the fourth week compared to pre-treatment sim-CT. Therefore, the most appropriate replanning time might be after 20 fractions of treatment. Besides, they also found pre-treatment body mass index (BMI) and tumor apparent diffusion coefficient (ADC) were potential predictive factors for the determination of replanning during IMRT.

In 2014, Li et al. (2014) analyzed local failure patterns for patients with NPC after IMRT. 710 patients with NPC were investigated.After a median follow-up of 38 months, 34 developed local recurrence. Because the original IMRT plans of two patients were lost, 32 were analyzed. The local failure patterns showed "central" in 16 patients, "marginal" in 9, and outside" in 7. In Kong et al's study (Kong et al., 2014), 370 patiens with NPC after IMRT were analyzed. With a median follow up of 26 months, localregional failure occurred in 25 patients. Among the 22 local-regional failures with available diagnostic images, 16 were in-field failures, 3 were marginal and 3 were outside-field failures. The main pattern for local-regional recurrence was in-field failure. Li et al. (2014) found the volumes of primary gross tumor and clinical target 1 were significantly correlated with recurrent patterns. For tumors with large volume, hypoxia in centre was more severe. Hong et al (2013) reviewed articles published on clinical and preclinical studies targeting tumor hypoxia. They found hypoxia was common in NPC and it was associated with disease progression and resistance to radiotherapy. Higher dose to the region of radiation resistance might reduce the pattern of in-field failure. Therefore, it was essential to develop the technology of radiation biology and image to help identify the the region of radiation resistance.

\section{Conclusions}

In conclusion, IMRT could provide higher Qol for loco-regionally advanced NPC. However, it needs to be addressed whether IMRT is superior to 2DRT in survival for loco-regionally advanced NPC in the mode of standard therapeutic regimen recommended by NCCN. Trials with large samples are essential to performed to determine the superiority of IMRT to 3DRT. Recent studies about helical tomotherapy has demonstrated its superiority in dose conformity. Because of some shortcomings in published studies regarding helical tomotherapy, it is urgent to conduct RCTs with large samples and long-term follow up to compare helical tomotherapy and traditional IMRT. There are great challenges to overcome in the future: (1) whether the addition of concurrent chemotherapy to IMRT and replanning are necessary; (2) updated delineation protocols regarding CTV and OAR are required; (3) Methods of identification of the region of radiation resistance are urgent; (4) the causes of the main local failure pattern are required to settle.

\section{References}

Chau RM, Teo PM, Kam MK, et al (2007). Dosimetric 
comparison between 2-dimensional radiation therapy and intensity modulated radiation therapy in treatment of advanced T-stage nasopharyngeal carcinoma: to treat less or more in the planning organ-at-risk volume of the brainstem and spinal cord. Med Dosim, 32, 263-70.

Chen AM, Yang CC, Marsano J, Liu T,Purdy JA (2012). Intensity-modulated radiotherapy for nasopharyngeal carcinoma: improvement of the therapeutic ratio with helical tomotherapy vs segmental multileaf collimator-based techniques. Br J Radiol, 85, 537-43.

Chen C, Lin X, Pan J, et al (2013). Is it necessary to repeat $\mathrm{CT}$ imaging and replanning during the course of intensitymodulated radiation therapy for locoregionally advanced nasopharyngeal carcinoma? Jpn J Radiol, 31, 593-9.

Du L, Zhang XX, Ma L, et al (2014). Clinical study of nasopharyngeal carcinoma treated by helical tomotherapy in china: 5-year outcomes. Biomed Res Int, 2014, 980767.

Fang FM, Chien CY, Tsai WL, et al (2008). Quality of life and survival outcome for patients with nasopharyngeal carcinoma receiving three-dimensional conformal radiotherapy $v s$ intensity-modulated radiotherapy-a longitudinal study. Int J Radiat Oncol Biol Phys, 72, 356-64.

Hong B, Lui VW, Hashiguchi M, Hui EP,Chan AT (2013). Targeting tumor hypoxia in nasopharyngeal carcinoma. Head Neck, 35, 133-45.

Hsiung CY, Ting HM, Huang HY, et al (2006). Parotidsparing intensity-modulated radiotherapy (IMRT) for nasopharyngeal carcinoma: Preserved parotid function after IMRT on quantitative salivary scintigraphy, and comparison with historical data after conventional radiotherapy. Int $J$ Radiat Oncol Biol Phys, 66, 454-61.

Jemal A, Bray F, Center MM, et al (2011). Global cancer statistics. CA Cancer J Clin, 61, 69-90.

Kong F, Ying H, Du C, et al (2014). Patterns of local-regional failure after primary intensity modulated radiotherapy for nasopharyngeal carcinoma. Radiat Oncol, 9, 60.

Lai SZ, Li WF, Chen L, et al (2011). How does intensitymodulated radiotherapy versus conventional twodimensional radiotherapy influence the treatment results in nasopharyngeal carcinoma patients? Int J Radiat Oncol Biol Phys, 80, 661-8.

Lee AW, Ng WT, Chan LL, et al (2014). Evolution of treatment for nasopharyngeal cancer--success and setback in the intensity-modulated radiotherapy era. Radiother Oncol, 110, 377-84.

Lee FK, Yip CW, Cheung FC, et al (2014). Dosimetric difference amongst 3 techniques: TomoTherapy, sliding-window intensity-modulated radiotherapy (IMRT), and RapidArc radiotherapy in the treatment of late-stage nasopharyngeal carcinoma (NPC). Med Dosim, 39, 44-9.

Li JX, Huang SM, Jiang XH, et al (2014). Local failure patterns for patients with nasopharyngeal carcinoma after intensitymodulated radiotherapy. Radiat Oncol, 9, 87.

Lin S, Pan J, Han L, et al (2009). Nasopharyngeal carcinoma treated with reduced-volume intensity-modulated radiation therapy: report on the 3-year outcome of a prospective series. Int J Radiat Oncol Biol Phys, 75, 1071-8.

Lin S, Lu JJ, Han L, Chen Q, Pan J (2010). Sequential chemotherapy and intensity-modulated radiation therapy in the management of locoregionally advanced nasopharyngeal carcinoma: experience of 370 consecutive cases. BMC Cancer, 10, 39.

Lin S, Pan J, Han L, et al (2014). Update report of nasopharyngeal carcinoma treated with reduced-volume intensity-modulated radiation therapy and hypothesis of the optimal margin. Radiother Oncol, 110, 385-9.

Lu J, Ma Y, Chen J, et al (2014). Assessment of anatomical and dosimetric changes by a deformable registration method during the course of intensity-modulated radiotherapy for nasopharyngeal carcinoma. J Radiat Res, 55, 97-104.

Mackie TR, Holmes T, Swerdloff S, et al (1993). Tomotherapy: a new concept for the delivery of dynamic conformal radiotherapy. Med Phys, 20, 1709-19.

Moretto F, Rampino M, Munoz F, et al (2014). Conventional 2D (2DRT) and 3D conformal radiotherapy (3DCRT) versus intensity-modulated radiotherapy (IMRT) for nasopharyngeal cancer treatment. Radiol Med, 119, 634-41.

Peng G, Wang T, Yang KY, et al (2012). A prospective, randomized study comparing outcomes and toxicities of intensity-modulated radiotherapy $v s$ conventional two-dimensional radiotherapy for the treatment of nasopharyngeal carcinoma. Radiother Oncol, 104, 286-93.

Phua Chee Ee V, Tan BS, Tan AL, et al (2013). Dose planning study of target volume coverage with intensity- modulated radiotherapy for nasopharyngeal carcinoma: Penang General Hospital experience. Asian Pac J Cancer Prev, 14, 2243-8.

Ren G, Du L, Ma L, et al (2011). Clinical observation of 73 nasopharyngeal carcinoma patients treated by helical tomotherapy: the China experience. Technol Cancer Res Treat, 10, 259-66.

Rong Y, Tang G, Welsh JS, et al (2011). Helical tomotherapy versus single-arc intensity-modulated arc therapy: a collaborative dosimetric comparison between two institutions. Int J Radiat Oncol Biol Phys, 81, 284-96.

Sun X, Su S, Chen C, et al (2014). Long-term outcomes of intensity-modulated radiotherapy for 868 patients with nasopharyngeal carcinoma: an analysis of survival and treatment toxicities. Radiother Oncol, 110, 398-403.

Sun Y, Guo R, Yin WJ, et al (2013). Which T category of nasopharyngeal carcinoma may benefit most from volumetric modulated arc therapy compared with step and shoot intensity modulated radiation therapy. PLoS One, $\mathbf{8}$, 75304.

Sun Y, Yu XL, Luo W, et al (2014). Recommendation for a contouring method and atlas of organs at risk in nasopharyngeal carcinoma patients receiving intensitymodulated radiotherapy. Radiother Oncol, 110, 390-7.

Tham IW, Hee SW, Yeo RM, et al (2009). Treatment of nasopharyngeal carcinoma using intensity-modulated radiotherapy-the national cancer centre singapore experience. Int J Radiat Oncol Biol Phys, 75, 1481-6.

Tsang RK, Kwong DL, Ho AC, et al (2012). Long-term hearing results and otological complications of nasopharyngeal carcinoma patients: Comparison between treatment with conventional two-dimensional radiotherapy and intensitymodulated radiotherapy. $O R L, \mathbf{7 4}, 228-33$.

Wiezorek T, Brachwitz T, Georg D, et al (2011). Rotational IMRT techniques compared to fixed gantry IMRT and tomotherapy: multi-institutional planning study for head-and-neck cases. Radiat Oncol, 6, 20.

Wu WC, Mui WL,Fung WK (2010). Helical tomotherapy of nasopharyngeal carcinoma-any advantages over conventional intensity-modulated radiotherapy? Med Dosim, 35, 122-7.

Yan D, Yan S, Wang Q, et al (2013). Predictors for replanning in loco-regionally advanced nasopharyngeal carcinoma patients undergoing intensity-modulated radiation therapy: a prospective observational study. BMC Cancer, 13, 548.

Yang H, Hu W, Wang W, et al (2013). Replanning during intensity modulated radiation therapy improved quality of life in patients with nasopharyngeal carcinoma. Int J Radiat Oncol Biol Phys, 85, 47-54.

Yi JL, Gao L, Huang XD, et al (2006). Nasopharyngeal carcinoma treated by radical radiotherapy alone: Ten-year 
Song Qu et al

experience of a single institution. Int J Radiat Oncol Biol Phys, 65, 161-8.

Zhang Y, Lin ZA, Pan JJ, et al (2009). Concurrent control study of different radiotherapy for primary nasopharyngeal carcinoma: Intensity-modulated radiotherapy versus conventional radiotherapy. Chin J Cancer, 28, 21-6. 